\title{
The effect of substrate on high-temperature annealing of GaN epilayers: Si versus sapphire
}

\author{
D. Pastor, R. Cuscó, and L. Artús ${ }^{\mathrm{a})}$ \\ Institut Jaume Almera, Consell Superior d'Investigacions Científiques (CSIC), Lluís Solé i Sabarís s.n., \\ 08028 Barcelona, Spain \\ G. González-Díaz \\ Departamento Física Aplicada III, Universidad Complutense, 28040 Madrid, Spain \\ E. Iborra \\ Departamento Tecnología Electrónica, ETSIT, Universidad Politécnica, 28040 Madrid, Spain \\ J. Jiménez \\ Departamento Física de la Materia Condensada, ETSII, Universidad de Valladolid, 47011 Valladolid, Spain \\ F. Peiró \\ Departamento Electrónica, Universidad de Barcelona, 08028 Barcelona, Spain \\ E. Calleja \\ ISOM, Universidad Politécnica, Ciudad Universitaria, 28040 Madrid, Spain and Departamento Ingeniería \\ Electrónica, ETSIT, Universidad Politécnica, Ciudad Universitaria, 28040 Madrid, Spain
}

(Received 6 February 2006; accepted 29 May 2006; published online 21 August 2006)

We have studied the effects of rapid thermal annealing at $1300{ }^{\circ} \mathrm{C}$ on $\mathrm{GaN}$ epilayers grown on AlN buffered $\mathrm{Si}(111)$ and on sapphire substrates. After annealing, the epilayers grown on Si display visible alterations with craterlike morphology scattered over the surface. The annealed $\mathrm{GaN} / \mathrm{Si}$ layers were characterized by a range of experimental techniques: scanning electron microscopy, optical confocal imaging, energy dispersive x-ray microanalysis, Raman scattering, and cathodoluminescence. A substantial Si migration to the $\mathrm{GaN}$ epilayer was observed in the crater regions, where decomposition of $\mathrm{GaN}$ and formation of $\mathrm{Si}_{3} \mathrm{~N}_{4}$ crystallites as well as metallic $\mathrm{Ga}$ droplets and Si nanocrystals have occurred. The average diameter of the Si nanocrystals was estimated from Raman scattering to be around $3 \mathrm{~nm}$. Such annealing effects, which are not observed in $\mathrm{GaN}$ grown on sapphire, are a significant issue for applications of $\mathrm{GaN}$ grown on $\mathrm{Si}(111)$ substrates when subsequent high-temperature processing is required. (c) 2006 American Institute of Physics. [DOI: 10.1063/1.2259817]

\section{INTRODUCTION}

Heteroepitaxy of GaN on $\mathrm{Si}(111)$ substrates is an interesting alternative for the fabrication of light emitting diodes (LEDs) and field effect transistors (FETs) because of the availability of large high-quality Si substrates at much lower cost than the sapphire and $\mathrm{SiC}$ substrates used for commercially available blue and green LEDs. Also, the development of a nitride-based optoelectronic technology on silicon is highly attractive for a possible integration with the mainstream $\mathrm{Si}(001)$ technology. ${ }^{1}$ A major problem for the growth of good quality $\mathrm{GaN}$ epilayers on $\mathrm{Si}$ is the large difference in thermal expansion coefficients, which in the cooling process often leads to cracked layers unsuitable for devices. Also, Si diffusion from the substrate ${ }^{2,3}$ and the formation of an amorphous $\operatorname{SiN}_{x}$ layer at the interface ${ }^{2}$ contribute to degrade the epilayer quality. The insertion of an AlN buffer is widely used to avoid these problems and to accommodate lattice mismatch. The realization of LED structures grown by molecular beam epitaxy (MBE) on $\mathrm{Si}(111)$ (Ref. 4) spurred the efforts on $\mathrm{GaN}$-based devices on $\mathrm{Si}$. In the last few years, there has been significant progress in the growth of high-

${ }^{\text {a)} E l e c t r o n i c ~ m a i l: ~ l a r t u s @ i j a . c s i c . e s ~}$ quality, thick GaN layers on Si. Several approaches to alleviate the thermal mismatch effects have been proposed. ${ }^{5}$ High-quality AlGaN/GaN heterostructures with twodimensional electron gas (2DEG) electron mobilities as large as $1800 \mathrm{~cm}^{2} / \mathrm{V}$ s have been obtained with good uniformity on $100 \mathrm{~mm} \mathrm{Si} \mathrm{substrates.}{ }^{6}$ Recently, a commercial company (Nitronex Inc.) has announced the release of a $50 \mathrm{~W}$ rf power transistor based on proprietary GaN on Si technology. The emission efficiency of GaN-based LED on Si is severely limited by the presence of cracks in the material and by high absorption losses in the GaN layer and in the Si substrate. Virtually crack-free LED structures were grown on $\mathrm{Si}$ by using low-temperature AlN interlayers and by in situ insertion of a $\mathrm{Si}_{x} \mathrm{~N}_{y}$ mask. ${ }^{7}$ Although blue LEDs obtained by this technique yielded an output power of $152 \mu \mathrm{W}$ at $20 \mathrm{~mA}$, which is sufficient for low-power signaling applications, the development of GaN-based LED devices on Si is still mainly restricted to academic research work.

Ion implantation is a doping technique specially suited to produce selective doping with high lateral definition and good control on the doping profile. The process introduces damage in the host lattice and a postimplantation thermal annealing step is required to restore crystallinity and activate 


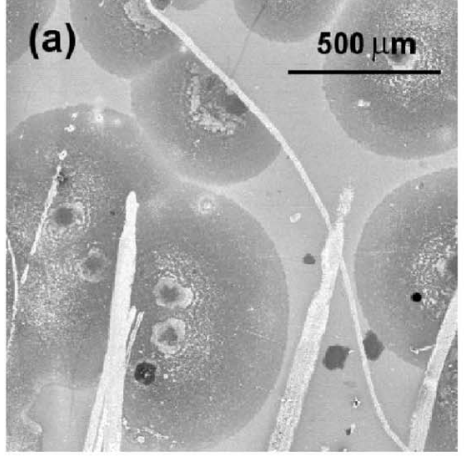

(b)

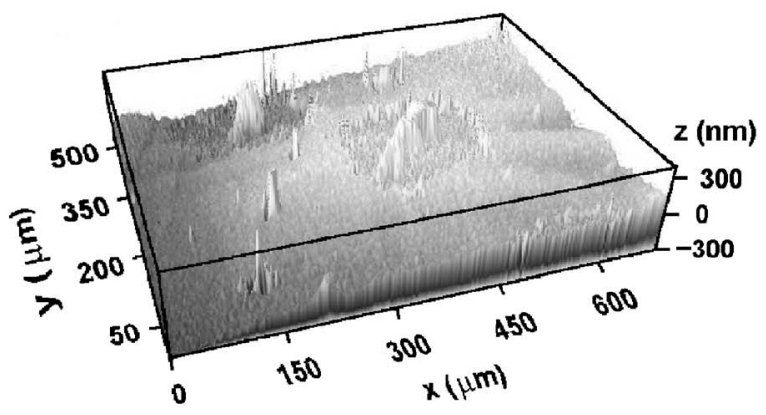

FIG. 1. (a) SEM image of the GaN epilayer on $\mathrm{Si}(111)$ after RTA at $1300{ }^{\circ} \mathrm{C}$ for $10 \mathrm{~s}$. (b) Topography of the damaged surface as revealed by confocal optical microscopy.

the dopants. In the case of GaN layers grown on $\mathrm{Si}$ substrate ion implantation doping may be problematic, since lattice recovery of implanted $\mathrm{GaN}$ is achieved only for annealing temperatures above $1200^{\circ} \mathrm{C}$. ${ }^{8} 9$ Such high annealing temperatures may have damaging effects on the $\mathrm{GaN} / \mathrm{Si}$ epilayers. In fact, decomposition of low-temperature-grown GaN on $\mathrm{Si}$ and corrosion of the $\mathrm{Si}$ substrate was observed after annealing at $1050{ }^{\circ} \mathrm{C},{ }^{10}$ and deterioration of the $\mathrm{GaN}$ layer by meltback etching is known to occur in $\mathrm{GaN}$ grown on $\mathrm{Si}$ at high temperatures. ${ }^{2}$

In the present work, we study the effects of hightemperature rapid thermal annealing (RTA) on GaN epilayers grown on AlN buffered $\mathrm{Si}(111)$ and on sapphire substrates. The annealing temperature used in the experiments $(T$ $=1300{ }^{\circ} \mathrm{C}$ ) is the highest temperature at which the encapsulating AlN layer is still effective, before the onset of AlN decomposition. ${ }^{9}$ Whereas the GaN epilayers grown on sapphire maintained their homogeneity and crystallinity after RTA, we found a severe degradation of the GaN epilayers grown on $\mathrm{Si}$ as a consequence of a substantial $\mathrm{Si}$ migration from the substrate. Since the damage removal for optimum electrical properties of implanted GaN requires very high annealing temperatures, the thermal instability of the Si substrate is a significant issue for ion-beam doping applications in GaN-based device technology on Si.

\section{EXPERIMENT}

The samples used in this study were GaN films of $\approx 1 \mu \mathrm{m}$ thickness grown by molecular beam epitaxy on $\operatorname{Si}(111)$ substrates after deposition of a thin $(\approx 20 \mathrm{~nm})$ AlN layer. GaN epilayers grown on sapphire were also studied for comparison purposes. To avoid surface decomposition of the GaN epilayer during high-temperature RTA, the samples were encapsulated with a reactively sputtered AlN film of $100 \mathrm{~nm}$, which was deposited in a Leybold Z-550 sputtering system.

The samples were annealed at $1300{ }^{\circ} \mathrm{C}$ for $10 \mathrm{~s}$ in a $80 \mathrm{~kW}$ ADDAX-RM infrared lamps reactor using a 4 in. diameter SiC susceptor. The samples were placed between the two plates of the susceptor with the Si substrate facing down in contact with the $\mathrm{SiC}$ holder plate. The temperature of the sample was monitored by means of a Pt thermocouple introduced through the susceptor holder plate in order to place its tip in close proximity to the sample. Energy dispersive x-ray (EDX) microanalysis was carried out in a Jeol JSM 6400 scanning electron microscope and the surface topography was investigated using a Sensofar confocal imaging profiler, with a depth resolution better than $5 \mathrm{~nm}$. Spectrally resolved cathodoluminescence (CL) measurements were carried out at $80 \mathrm{~K}$ with a XiCLone monoCL3 system from Gatan. MicroRaman measurements were performed using a Jobin-Yvon T64000 spectrometer. The excitation source was the $514.5 \mathrm{~nm}$ line of an $\mathrm{Ar}^{+}$laser and the measurements were done in backscattering configuration with spatial resolution of $\approx 1 \mu \mathrm{m}$. Focused ion-beam (FIB) milling experiments were performed in an FEI dual beam DB232 Strata system, using $\mathrm{Ga}$ ions accelerated at $30 \mathrm{kV}$ and ion-beam currents in the $1-20 \mathrm{nA}$ range.

\section{RESULTS AND DISCUSSION}

Figure 1(a) shows a scanning electron microscopy (SEM) image of the GaN epilayer on $\mathrm{Si}(111)$ after RTA at $1300{ }^{\circ} \mathrm{C}$ for $10 \mathrm{~s}$. Coalescent dark regions that develop around the nuclei of typically $50 \mu \mathrm{m}$ diameter can be observed all over the sample surface. As can be seen in the confocal optical microscopy image shown in Fig. 1(b), the sample surface exhibits a high degree of roughness, with height variations of the order of several hundreds of nanometers, and craterlike regions can be observed around the protruding nuclei. Element analysis carried out by EDX revealed a substantial presence of $\mathrm{Si}$ (around 30\% atomic concentration) in the central nuclei as well as a strong depletion of $\mathrm{Ga}$ and nonstoichiometric $\mathrm{Ga} / \mathrm{N}$ ratios of about 1:4. The $\mathrm{Si}$ atomic concentration was found to be consistently high over the dark regions, with values around $20 \%$, whereas it dropped below detection levels in the homogeneous regions in between. These results suggest that a massive $\mathrm{Si}$ migration from the substrate takes place at specific points of the epilayer during the high-temperature annealing. Si migration from the substrate to the surface had been previously observed in GaN layers directly grown on Si by MBE (Ref. 3 ) and by metal-organic vapor phase epitaxy. ${ }^{2}$ The mechanism for the anomalously high $\mathrm{Si}$ migration observed in the $\mathrm{GaN} / \mathrm{Si}$ samples annealed at $1300{ }^{\circ} \mathrm{C}$ is not well understood yet, but it could be related to the columnar structure of heteroepitaxial $\mathrm{GaN}$ layers ${ }^{11}$ and to the presence of grain boundaries. Pipe diffusion through threading dislocations ${ }^{12}$ may also enhance Si migration, which due to the strong reactivity of $\mathrm{Ga}$ and $\mathrm{Si}$ at high temperatures gives rise to the meltback etching effect. 2,10

To further characterize the material alterations in the annealed GaN epilayer grown on $\mathrm{Si}$, micro-Raman measure- 

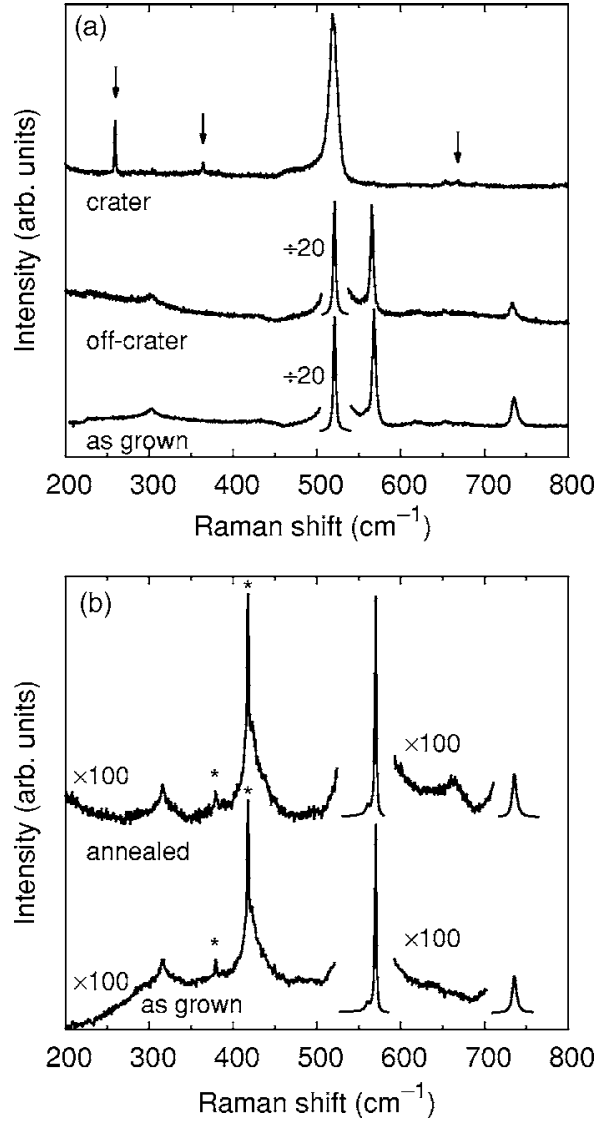

FIG. 2. (a) Raman spectra of GaN epilayers grown on $\mathrm{Si}(111)$ after RTA obtained in the crater region and from homogeneous regions between craters, compared with the spectrum of the layers before annealing. The arrows indicate Raman peaks corresponding to crystalline $\mathrm{Si}_{3} \mathrm{~N}_{4}$. (b) Raman spectra obtained from $\mathrm{GaN}$ epilayers grown on sapphire before and after RTA under identical conditions. The peaks marked with an asterisk correspond to the sapphire substrate.

ments were carried out in the crater area and in the homogeneous region between craters. In Fig. 2(a) we compare the Raman spectra of the annealed sample with the spectrum obtained before annealing. In addition to the strong Raman peak at $521 \mathrm{~cm}^{-1}$ arising from the $\mathrm{Si}$ substrate, the characteristic $E_{2}$ and $A_{1}(\mathrm{LO})$ peaks of $\mathrm{GaN}$ can be observed, respectively, at 568 and $735 \mathrm{~cm}^{-1}$ in the spectra of both the as-grown sample and the homogenous off-crater region. No appreciable changes in the intensity and width of the $E_{2}$ peaks are found, which indicate that the homogeneous regions retain a good crystallinity after annealing. The lower intensity and slight broadening exhibited by the $A_{1}(\mathrm{LO})$ mode in the annealed layer can be attributed to an increased density of free carriers due to the diffusion of Si donor atoms, which to a much lower degree also takes place in the crater-free regions. ${ }^{3,13,14}$ In contrast, the Raman spectrum of the crater region is dramatically changed. No Raman signal of the GaN modes can be detected in these regions, which display a Raman spectrum dominated by a considerably broadened Si peak. Additional sharp peaks emerge at 260, 364 , and $668 \mathrm{~cm}^{-1}$ that are in good agreement with the reported Raman frequencies of $\alpha-\mathrm{Si}_{3} \mathrm{~N}_{4}{ }^{15}$ The detection of these modes clearly indicate that in the crater regions the $\mathrm{Si}$ migrating from the substrate strongly reacts with GaN giving

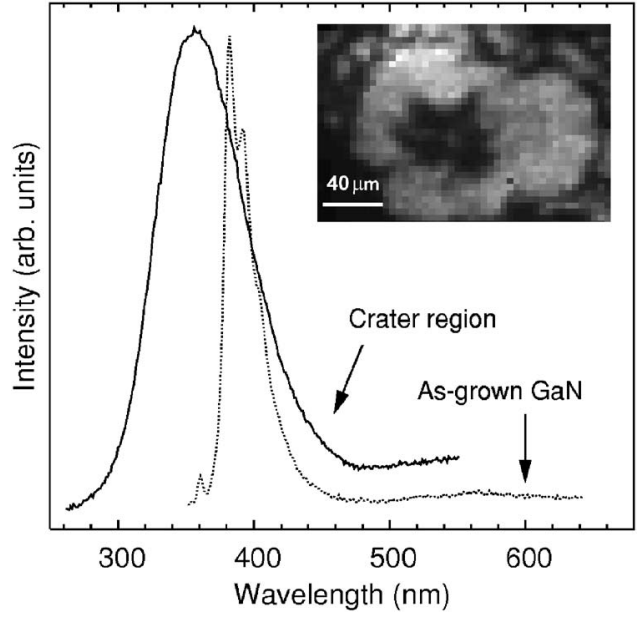

FIG. 3. Cathodoluminescence spectra of $\mathrm{GaN} / \mathrm{Si}$ epilayers obtained at $T$ $=80 \mathrm{~K}$ from the crater region of an annealed sample (solid line) and from a homogeneous sample without annealing (dotted line). The spectra have been normalized to display the same maximum intensity. Inset, cathodoluminescence mapping of the crater region with wavelength detection set at $350 \mathrm{~nm}$.

rise to $\alpha-\mathrm{Si}_{3} \mathrm{~N}_{4}$ crystallites. This is in contrast with previous reports on the formation of amorphous $\mathrm{SiN}_{x}$ in direct epitaxial growth of $\mathrm{GaN}$ on $\mathrm{Si}$ at growth temperatures lower than the annealing temperature used in this study. The high annealing temperature used for the RTA cycle favors the crystallization of $\alpha-\mathrm{Si}_{3} \mathrm{~N}_{4}$ with good crystallinity, as evidenced by the sharp peaks observed in the Raman spectrum of Fig. 2(a). Also, since Ga is highly corrosive to the Si substrate, ${ }^{10}$ the latter is degraded by meltback effect in the crater regions as indicated by the broadening of the Si Raman peak.

For comparison, the same annealing study was carried out on GaN layers grown on sapphire. In Fig. 2(b) we display the Raman spectra of the GaN/sapphire epilayers before and after RTA at $1300{ }^{\circ} \mathrm{C}$ for $10 \mathrm{~s}$. Both spectra are dominated by the $E_{2}$ and $A_{1}(\mathrm{LO})$ peaks, which do not show any noticeable change in intensity and width after annealing. The only distinctive feature that can be observed in the Raman spectrum after RTA is a weak band emerging between the $E_{2}$ and the $A_{1}(\mathrm{LO})$ frequencies. This band was previously observed in ion-beam implanted GaN layers and was assigned to vacancy-related defects. ${ }^{16,17}$ In contrast with the case of $\mathrm{GaN} / \mathrm{Si}(111)$, the $A_{1}(\mathrm{LO})$ peak maintains its intensity after annealing, indicating that the residual free carrier concentration has not appreciably changed in GaN/sapphire after RTA. Even the forbidden $E_{1}(\mathrm{TO})$ that can be seen as a weak feature at $\approx 560 \mathrm{~cm}^{-1}$ in the low energy side of the $E_{2}$ peak displays the same intensity after RTA. These results show that, contrary to the $\mathrm{GaN} / \mathrm{Si}(111)$ case, the annealing process does not alter the crystalline quality of the GaN epilayers grown on sapphire.

The annealing effects on the GaN/Si epilayers have also been characterized by spectrally resolved CL measurements. In Fig. 3(a) we compare a CL spectrum obtained at $80 \mathrm{~K}$ in the crater region of a GaN/Si annealed sample (solid line) with a typical GaN CL spectrum of an unannealed sample (dotted line). Several bands are resolved in the CL spectrum of the unannealed sample. The weak feature at $360 \mathrm{~nm}$ has been assigned to a donor bound exciton, whereas the peaks at 

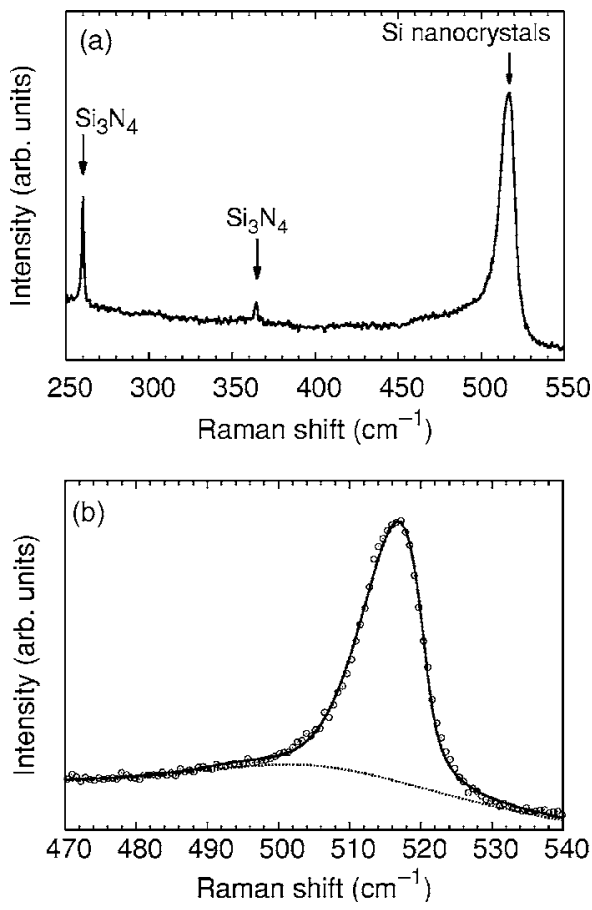

FIG. 4. (a) Raman spectrum obtained in the crater region displaying both peaks corresponding to $\mathrm{Si}_{3} \mathrm{~N}_{4}$ crystallites and a redshifted $\mathrm{Si}$ peak arising from Si nanocrystals. (b) Fit of the phonon confinement model (solid line) to the Si Raman peak (circles). An amorphouslike broad band contribution (dotted line) has also been included in the model.

382 and $391 \mathrm{~nm}$ are due to a donor-acceptor pair recombination and its corresponding phonon replica. ${ }^{18}$ In contrast, the CL spectrum obtained in the crater region displays only a broad band that is clearly blueshifted in relation to the near band gap emission of GaN. The inset of Fig. 3(a) shows a CL image of the crater region obtained for an emission wavelength of $350 \mathrm{~nm}$. A bright halo is observed around the nonemitting core. Taking into account that the Raman spectra obtained in these regions show no Raman signal from GaN and clear peaks of $\mathrm{Si}_{3} \mathrm{~N}_{4}$, we believe that this emission may be related to the formation of $\mathrm{Si}_{3} \mathrm{~N}_{4}$. Given that the $\mathrm{Si}_{3} \mathrm{~N}_{4}$ band gap is $4.6 \mathrm{eV},{ }^{19}$ the lower emission energy of the CL peak as well as its large width suggest band gap fluctuations and the formation of potential wells due to the presence of $\mathrm{SiN}_{x}$ nanoclusters of nonuniform composition. ${ }^{19}$ A detailed Raman scattering study of the crater region reveals indeed the existence of Si nanocrystals, as indicated by strong redshifts of the Si Raman peak due to phonon confinement. ${ }^{20}$ In Fig. 4(a) we show a Raman spectrum obtained in the crater region where, besides the Raman peaks of crystalline $\mathrm{Si}_{3} \mathrm{~N}_{4}$, a broadened Si peak of reduced intensity can be observed at $517 \mathrm{~cm}^{-1}$. In contrast with the Raman spectrum of the homogeneous region shown in Fig. 2, which was dominated by the Si Raman peak of the substrate, no Raman signal of the bulk $\mathrm{Si}$ substrate is detected in this spectrum, probably due to absorption in the altered material and to a locally stronger meltback etching at the probed location. We attribute the strong redshift of the Si Raman peak to phonon confinement effects in the Si nanocrystals that form as a result of the strong Si migration during RTA. To evaluate the size of the Si nanocrystals, we have fitted our Raman data using the spatial correlation model (SCM). ${ }^{20-22}$ We follow the approach proposed by Paillard et al., ${ }^{22}$ in which the strong anisotropy of the Si phonon band dispersion was taken into account using Brout's sum rule to determine an average optical mode band dispersion as

$$
\bar{\omega}_{\text {opt }}(\mathbf{q})=\sqrt{\omega_{0}^{2}-\frac{1.261 \times 10^{5} q^{2}}{q+0.53}} \quad(q<0.5),
$$

where $\omega_{0}$ is the zone-center optical phonon frequency of $\mathrm{Si}$ and $q$ is the reduced wave vector expressed in units of $2 \pi / a$, with $a$ the lattice parameter. The line shape of phonons confined to a hard sphere of diameter $D$ is then given by

$$
\mathcal{L}(\omega ; D) \propto \int_{0}^{0.5} d q \frac{\sin ^{2}[(q D / a) \pi]}{\left[1-(q D / a)^{2}\right]^{2}} \frac{1}{\left[\bar{\omega}_{\mathrm{opt}}(q)-\omega\right]^{2}+(\Gamma / 2)^{2}},
$$

where $\Gamma$ is the damping parameter of the optical phonon of Si. The use of the SCM [Eq. (2)] with the averaged phonon dispersion given by Eq. (1) has been shown to yield phonon confinement shifts in good agreement with more elaborate microscopic calculations based on the partial density approach and the bond polarizability model, ${ }^{21,22}$ and has been successfully applied to determine crystallite diameters in nanocrystalline $\mathrm{Si}$ films. ${ }^{22}$ One should note the validity range of Eq. (1), which limits the applicability of the model to crystallite diameters $D>2.2 \mathrm{~nm}$.

We have fitted Eq. (2) to our Raman data on annealed GaN/Si layers. We used $\omega_{0}=521 \mathrm{~cm}^{-1}$ and $\Gamma=3 \mathrm{~cm}^{-1}$, as obtained from Raman measurements on bulk Si corrected for the spectral resolution of the spectrometer. In Fig. 4(b) we show the calculated line shape for $D=2.7 \mathrm{~nm}$, where a broad band (dotted line) was also included in the fit to account for scattering by modes of the oxidized surface of the nanocrystals. ${ }^{22}$ As can be seen in Fig. 4(b), an excellent fit is obtained to both the frequency and the width of the experimental spectrum. Since both frequency shift and broadening are completely determined by the nanocrystal diameter in the SCM model, this indicates that the observed frequency shift is entirely accounted for by phonon confinement effects and therefore strain effects are negligible in this case. The obtained $D$ value represents an average crystallite diameter. This was found to be fairly homogeneous over the crater regions, where fits to Raman spectra obtained at different locations consistently give $D$ values around $3 \mathrm{~nm}$.

The decomposition of $\mathrm{GaN}$ by chemical reaction with $\mathrm{Si}$, which as already discussed yields $\mathrm{Si}_{3} \mathrm{~N}_{4}$ crystallites, results also in the formation of metallic Ga droplets. ${ }^{10}$ This was strikingly demonstrated by carrying out an ion-beam milling of an $8 \times 4 \mu \mathrm{m}^{2}$ area in the crater region, which removed the host material in the milled area and allowed the coalescence of the metallic Ga droplets. Figure 5 shows SEM images of the sample at different stages of the etching process. As the milling proceeded to a depth of $\approx 1 \mu \mathrm{m}$, the etched cavity was observed to fill with metallic Ga, which having a melting point of nearly $30{ }^{\circ} \mathrm{C}$ is readily liquified by the increase in sample temperature caused by the ion-beam bombardment. This is illustrated in Fig. 5, where we display SEM images of the etched cavity after $16 \mathrm{~s}$ (a) and $48 \mathrm{~s}$ (b) of FIB 

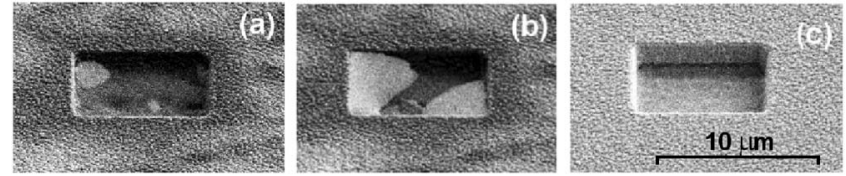

FIG. 5. SEM images of an $8 \times 4 \mu \mathrm{m}^{2}$ ion-milled area in a crater region of the annealed sample taken after $16 \mathrm{~s}$ (a) and $48 \mathrm{~s} \mathrm{(b)} \mathrm{of} \mathrm{ion-beam} \mathrm{etching}$ showing metallic Ga flooding the bottom of the cavity. Panel (c) shows the clear-cut walls obtained by ion-beam milling in the homogenous region between craters.

etching, the latter corresponding to a milling depth of $\approx 1 \mu \mathrm{m}$. The lighter areas observed at either side of the milled rectangle correspond to liquid Ga drops starting to fill up the bottom of the cavity. These showed a temporal evolution and after $60 \mathrm{~s}$ they eventually coalesced to cover completely the bottom of the milled cavity. This effect was observed for beam currents as low as $1 \mathrm{nA}$. By contrast, no metallic Ga could be observed in an equivalent cavity milled in the homogeneous region between craters, even though a beam current of $20 \mathrm{nA}$ was used in this case. Figure 5(c) shows a cavity milled in the homogeneous region, displaying clear-cut walls and homogeneous dark base surface. Unfortunately, the Ga coalescence effect precluded the preparation of thinned samples from the crater region and transmission electron microscopy measurements could not be carried out.

\section{CONCLUSION}

We have shown that the high-temperature annealing behavior of GaN epilayers depends critically on the substrate. Whereas GaN samples grown on sapphire can undergo RTA cycles to $1300{ }^{\circ} \mathrm{C}$ without significant crystalline degradation, severe alterations of the epilayer are observed in $\mathrm{GaN}$ samples grown on $\mathrm{Si}(111)$. Substantial migration of $\mathrm{Si}$ from the substrate to the epilayer surface has been detected at specific locations of the sample where craters are formed. In these regions, the strong reactivity between $\mathrm{Ga}$ and $\mathrm{Si}$ gives rise to the decomposition of $\mathrm{GaN}$ with the formation of $\mathrm{Si}_{3} \mathrm{~N}_{4}$ crystallites, metallic Ga droplets, and Si nanocrystals. The SCM successfully accounts for the observed frequency shift and broadening of the optical phonon modes of the $\mathrm{Si}$ nanocrystals, and it allows us to estimate an average size of around $3 \mathrm{~nm}$ for these nanocrystals. To a much lower degree, some $\mathrm{Si}$ diffusion from the substrate also takes place in the homogeneous regions between craters during RTA, which gives rise to an increase of the residual free-electron concentration of the layer. The deterioration of the $\mathrm{GaN} / \mathrm{Si}(111)$ epilayers observed in samples with thin AlN buffers is a significant problem that has to be addressed in device applications when processes that necessarily rely on subsequent high-temperature annealing cycles are involved.

\section{ACKNOWLEDGMENTS}

This work has been supported by the Spanish Ministry of Science and Technology under Contract Nos. MAT20040664 and MAT2004-02875. The authors wish to thank Dr. M. Marsal for her expert assistance with the EDX measurements.

${ }^{1}$ F. Schulze, A. Dadgar, J. Bläsing, and A. Krost, J. Cryst. Growth 272, 496 (2004).

${ }^{2}$ A. Krost and A. Dadgar, Mater. Sci. Eng., B 93, 77 (2002).

${ }^{3}$ E. Calleja et al., Phys. Rev. B 58, 1550 (1998).

${ }^{4}$ S. Guha and N. A. Bojarczuk, Appl. Phys. Lett. 72, 415 (1998).

${ }^{5}$ A. Krost and A. Dadgar, Phys. Status Solidi A 194, 361 (2002).

${ }^{6}$ J. D. Brown, R. Borges, E. Piner, A. Vescan, S. Singhal, and R. Therrien, Solid-State Electron. 46, 1535 (2002).

${ }^{7}$ A. Dadgar, M. Poschenrieder, J. Bläsing, K. Feshe, A. Diez, and A. Krost, Appl. Phys. Lett. 80, 3670 (2002).

${ }^{8}$ S. O. Kucheyev, J. S. Williams, and S. J. Pearton, Mater. Sci. Eng., R. 33, $51(2001)$.

${ }^{9}$ H. H. Tan, J. S. Williams, J. Zou, D. J. H. Cockayne, S. J. Pearton, J. C. Zolper, and R. A. Stall, Appl. Phys. Lett. 72, 1190 (1998).

${ }^{10}$ H. Ishikawa, K. Yamamoto, T. Egawa, T. Soga, T. Jimbo, and M. Umeno, J. Cryst. Growth 189/190, 178 (1998).

${ }^{11}$ E. Calleja et al., J. Cryst. Growth 201/202, 296 (1999).

${ }^{12}$ A. Gutiérrez-Sosa, U. Bangert, A. J. Harvey, C. J. Fall, R. Jones, P. R. Briddon, and M. I. Heggie, Phys. Rev. B 66, 035302 (2002).

${ }^{13}$ T. Kozawa, T. Kachi, H. Kano, Y. Taga, M. Hashimoto, N. Koide, and K. Manabe, J. Appl. Phys. 75, 1098 (1994).

${ }^{14}$ F. A. Ponce, J. W. Steeds, C. D. Dyer, and G. D. Pitt, Appl. Phys. Lett. 69, 2650 (1996).

${ }^{15}$ T. Kuzuba, K. Kijima, and Y. Brando, J. Chem. Phys. 69, 40 (1978).

${ }^{16}$ W. Limmer, W. Ritter, R. Sauer, B. Mensching, C. Liu, and B. Rauschenbach, Appl. Phys. Lett. 72, 2589 (1998).

${ }^{17}$ D. Pastor, R. Cuscó, L. Artús, G. González-Díaz, S. Fernández, and E. Calleja, Semicond. Sci. Technol. 20, 374 (2005).

${ }^{18}$ M. Toth, K. Fleischer, and M. R. Phillips, Phys. Rev. B 59, 1575 (1999).

${ }^{19}$ V. A. Gritsenko, K. S. Zhuravlev, A. D. Milov, H. Wong, R. W. M. Kwok, and J. B. Xu, Thin Solid Films 353, 20 (1999).

${ }^{20}$ H. Richter, Z. P. Wang, and L. Ley, Solid State Commun. 39, 625 (1981).

${ }^{21}$ J. Zi, H. Büscher, C. Falter, W. Ludwig, K. Zhang, and X. Xie, Appl. Phys. Lett. 69, 200 (1996).

${ }^{22}$ V. Paillard, P. Puech, M. A. Laguna, R. Carles, B. Kohn, and F. Huisken, J. Appl. Phys. 86, 1921 (1999). 